Reprod. Nutr. Dévelop., 1983, 23 (2 A), 195-206.

\title{
Influence du cholestérol et de la nature des acides gras alimentaires sur les activités de quelques monoxygénases microsomales du foie chez le rat
}

\author{
Marie-Hélène SIESS, Marie-France VERNEVAUT, M. SUSCHETET \\ Station de Recherches sur la Qualité des Aliments de l'Homme, \\ I.N.R.A., 7, rue Sully, 21034 Dijon Cedex.
}

Summary. The effect of cholesterol and the nature of dietary fatty acids on the activities of some microsomal monooxygenases in rat liver.

For 9 weeks, 2 -month old male rats were fed diets containing 0 or $2 \%$ cholesterol and $15 \%$ corn oil or coconut oil $(2 \times 2$ factorial experiment). The activity of several cytochrome P450-dependent monooxygenases from liver microsomes was measured. Diet ary cholesterol increased liver weight; this effect was more marked in rats fed unsaturated fat than in those fed saturated fat. The activity of NADPH-cytochrome c reductase and cytochrome $b_{5}$ contents was also enhanced by dietary cholesterol. The type of dietary fat mainly affected the activities of NADPH-cytochrome c reductase and aminopyrine demethylase. The latter was $34 \%$ higher in rats fed corn oil than in rats fed coconut oil. In contrast, the activities of aniline hydroxylase and laurate hydroxylase were not modified by dietary fat. Briefly, the dietary factors studied affected the activity of microsomal monooxygenases differently.

\section{Introduction.}

Les effets des lipides alimentaires, d'un point de vue aussi bien qualitatif que quantitatif, sur les activités d'enzymes microsomales, ont retenu l'attention de nombreux chercheurs depuis quelques années. Les lipides représentent en effet $30 \%$ à $40 \%$ du poids sec du réticulum endoplasmique et sont constitués pour une large part de phospholipides (50\% à $70 \%$ ) et de $7 \%$ à $10 \%$ de cholestérol (Glauman et Dallner, 1968). Or la composition en acides gras des phospholipides de ces membranes, ainsi que leur teneur en cholestérol, dépendent de la nature de l'apport lipidique alimentaire (Hammer et Wills, 1979 ; Laitinen, 1976 ; Spector et al., 1980). Ces modifications de constitution des microsomes entraînent des modifications de l'activité des enzymes qui y sont liées. Ainsi les activités de monoxygénases et les teneurs en cytochrome $\mathrm{P} 450$ de rats soumis à un régime lipidoprive sont inférieures à celles de rats nourris avec un régime comportant des lipides. Les huiles riches en acides gras insaturés permettent un meilleur 
fonctionnement des monoxygénases microsomales que les huiles riches en acides gras saturés (Century, 1973; Rowe et Wills, 1976 ; Wade et Norred, 1976). Ces différences ont été attribuées en partie au taux d'acide linoléique des différents types d'huile (Caster et al., 1970 ; Marshall et McLean, 1971). Cependant, d'autres constituants lipidiques du régime, principalement le cholestérol, ont un effet stimulant sur les activités de monoxygénases dépendantes du cytochrome P450 (Hietanen et al., 1978 ; Lambert et Wills, 1977 ; Lang et al., 1976).

Dans ce travail, il nous a donc paru intéressant d'étudier l'influence du cholestérol associé à deux types de corps gras alimentaires différents, I'un principalement saturé (huile de coprah), l'autre principalement insaturé (huile de maïs) sur l'activité de quelques enzymes microsomales dépendantes du cytochrome P450. Le dispositif expérimental que nous avons mis en place nous a permis d'étudier conjointement les effets dus à la nature de l'huile ingérée, ceux dus à la présence ou l'absence de cholestérol et ceux dus à l'interaction entre ces deux facteurs.

\section{Matériel et méthodes.}

1) Animaux d'expérience et régimes. - Trente-deux rats mâles Wistar EOPS sont nourris pendant deux mois avec une provende commerciale (UAR 113) renfermant environ $5 \%$ de lipides. Puis ils sont répartis en quatre lots de 8 rats, placés en cages individuelles et nourris avec des régimes semisynthétiques dont la composition est indiquée dans le tableau 1. L'huile de coprah utilisée est additionnée d'huile de tournesol dans un rapport $9 / 1$, de façon à fournir un apport en acide linoléique suffisant. Eau et régime sont donnés ad libitum. La consommation de nourriture est mesurée quotidiennement tout au long de l'expérience. On pèse les rats individuellement tous les 3 jours. Les animaux sont sacrifiés au bout de 9 semaines de prise de régime expérimental.

2) Préparation de la fraction microsomale hépatique. - Après un jeûne de $18 \mathrm{~h}$, les animaux sont sacrifiés et les foies immédiatement prélevés puis pesés. Ils sont homogénéisés au moyen d'un Potter, dans 3 volumes de saccharose $0,25 \mathrm{M}$ à $4{ }^{\circ} \mathrm{C}$. Les homogénats obtenus sont ensuite centrifugés à $10000 \mathrm{~g}$ pendant $20 \mathrm{~min}$. Une partie aliquote des surnageants est alors centrifugée à $105000 \mathrm{~g}$ pendant $90 \mathrm{~min}$. Les culots de microsomes sont lavés par un tampon Tris- $\mathrm{HCl} 0,15 \mathrm{M}, \mathrm{pH} 8$, puis centrifugés à nouveau à $105000 \mathrm{~g}$. Les microsomes sont enfin mis en suspension dans du saccharose $0,25 \mathrm{M}$ à la concentration d'environ 12 à $14 \mathrm{mg}$ de protéines par $\mathrm{ml}$ et conservés à ${ }^{\circ}-60^{\circ} \mathrm{C}$.

3) Dosages des protéines et du cholestérol. - Les concentrations en protéines des différentes fractions microsomales sont déterminées selon la méthode de Lowry et al. (1951). Le cholestérol est extrait, après saponification dans de la potasse alcoolique, par de l'éther de pétrole et dosé selon Abell et al. (1952).

4) Déterminations des activités enzymatiques. - Les teneurs en cytochrome P450 et $b_{5}$ sont déterminées selon Omura et Sato (1964).

La mesure de l'activité de la NADPH-cytochrome $c$ réductase est effectuée 
TABLEAU 1

Composition des régimes expérimentaux $(\mathrm{g})$ et composition en acides gras des deux types d'huile (\% des acides gras totaux)

\begin{tabular}{lcccc}
\hline \multicolumn{1}{c}{ Composants } & Maïs & $\begin{array}{c}\text { Maïs }+ \\
\text { cholestérol }\end{array}$ & Coprah & $\begin{array}{c}\text { Coprah + } \\
\text { cholestérol }\end{array}$ \\
\hline Caséine & 23 & 23 & 23 & 23 \\
Amidon & 32 & 32 & 32 & 32 \\
Saccharose & 24 & 22 & 24 & 22 \\
Mélange salin (1) & 4 & 4 & 4 & 4 \\
Solution vitaminique $\left(^{2}\right)$ & 1 & 1 & 1 & 1 \\
Huile de maïs & 15 & - & - & 15 \\
Huile de coprah & - & 2 & - & 2 \\
Cholestérol (3) & - & 2 & 2 & 2 \\
Agar & 2 & 199 & 199 & 199 \\
Eau & 199 & & & \\
\hline
\end{tabular}

Composition en acides gras

\begin{tabular}{lrrr}
$C 8: 0$ & & & \\
C $10: 0$ & & - & 8,3 \\
C $12: 0$ & & 7,4 \\
C $14: 0$ & & - & 45,0 \\
C $16: 0$ & & & 15,7 \\
C $18: 0$ & & 11,5 & 8,4 \\
C $18: 1$ & & 1,9 \\
C $18: 2$ & & & 6,4 \\
\hline
\end{tabular}

(1) Mélange de Hubbel et al. (1937) à ces différences près (en $\mathrm{g}$ par $\mathrm{kg}$ de mélange salin): $\mathrm{MnSO}_{4}, \mathrm{H}_{2} \mathrm{O} 3,50 ; \mathrm{ZnCl}_{2} 1,66 ; \mathrm{CoCl}_{2}, 6 \mathrm{H}_{2} \mathrm{O} 0,08$.

(2) La solution vitaminique apporte pour $1 \mathrm{~kg}$ de régime sec : vitamine $A$ hydrosoluble $6000 \mathrm{UI}$, vitamine $D_{2} 500 \mathrm{UI}$, vitamine $E$ hydrosoluble $100 \mathrm{mg}$, vitamine $K_{1} 1 \mathrm{mg}$, riboflavine $4 \mathrm{mg}$, vitamine $B_{6}$ $6 \mathrm{mg}$, acide pantothénique $10 \mathrm{mg}$, amide nicotinique $50 \mathrm{mg}$, acide folique $1 \mathrm{mg}$, biotine $0,2 \mathrm{mg}$, vitamine $B_{12} 0,03 \mathrm{mg}$, thiamine $4 \mathrm{mg}$, chlorure de choline $1000 \mathrm{mg}$, inositol $200 \mathrm{mg}$, acide p-aminobenzoïque $500 \mathrm{mg}$.

${ }^{\left({ }^{3}\right)}$ Le cholestérol est dissous dans l'huile avant d'être incorporé dans le régime.

d'après Phillips et Langdon (1962) à $20^{\circ} \mathrm{C}$. On détermine pendant 3 min l'amplitude de la variation de la densité optique à $550 \mathrm{~nm}$. La concentration en protéines dans l'essai est de $0,05 \mathrm{mg} / \mathrm{ml}$. L'activité de la NADH-cytochrome c réductase est évaluée dans les mêmes conditions en utilisant une concentration de $300 \mu \mathrm{M}$ de $\mathrm{NADH}$.

Les activités de l'aminopyrine $\mathrm{N}$-déméthylase et de l'aniline hydroxylase, sont mesurées après incubation de ces deux substrats pendant $20 \mathrm{~min}$, à $37^{\circ} \mathrm{C}$, sous agitation dans un milieu renfermant: tampon phosphate $83,5 \mathrm{mM}$ à $\mathrm{pH}=7,4 ; \mathrm{MgCl}_{2}: 6,66 \mathrm{mM}$; glucose-6-phosphate : $2,5 \mathrm{mM}$; NADP : $1 \mathrm{mM}$; glucose-6-phosphate déshydrogénase 1,2 UI/ $\mathrm{ml}$. Le volume final est de $5 \mathrm{ml}$ et la quantité de protéines microsomales de $5 \mathrm{mg}$. Les concentrations en substrat sont de $5 \mathrm{mM}$ pour l'aminopyrine et de $10 \mathrm{mM}$ pour l'aniline. La réaction est stoppée par addition de TCA. L'activité de l'aminopyrine N-déméthylase est appréciée par dosage de la 4-aminoantipyrine (Gilbert et Golberg, 1965) et celle de l'aniline hydroxylase par dosage du p-aminophénol (Imai et al., 1966). 
L'activité de la laurate hydroxylase est mesurée selon Salaün et al. (1981) en utilisant une concentration en laurate de sodium de $0,1 \mathrm{mM}$ et une quantité de protéines de 2 à $3 \mathrm{mg}$ par essai.

5) Analyses statistiques. - Les 4 lots expérimentaux sont disposés selon un plan factoriel $2^{2}$ ( 2 facteurs à 2 niveaux, nature de l'huile - coprah ou maïs - et présence ou non de cholestérol). Ce plan permet après analyse de variance, d'effectuer une décomposition orthogonale et par la méthode des contrastes (Dagnélie, 1973) de tester l'effet principal de chaque facteur ainsi que l'interaction éventuelle entre les deux, en utilisant le F de Fisher Snedecor. Dans le cas d'une interaction significative des comparaisons groupe à groupe sont effectuées.

\section{Résultats.}

1) Poids des animaux et poids relatifs du foie en fin d'expérimentation. Les régimes n'ont pas d'influence sur les poids des animaux en fin d'expérimentation (tabl. 2). Par contre, les poids relatifs des foies sont modifiés par l'apport de cholestérol alimentaire. Dans le cas du régime à base d'huile de coprah, on observe une augmentation de $22 \%$ et, dans le cas du régime à base d'huile de maïs, une augmentation de $53 \%$. L'effet de l'huile sur les poids relatifs des foies n'est observable que lorsque le cholestérol est présent dans le régime. Dans ce cas, les poids relatifs sont supérieurs de $20 \%$ pour le régime contenant de l'huile de maïs par rapport au régime contenant de l'huile de coprah.

\section{TABLEAU 2}

Poids des animaux et poids relatifs des foies en fin d'experimentation (moyennes et écart-type commun, 8 rats/lot)

\begin{tabular}{lcc}
\hline \multicolumn{1}{c}{ Traitements } & $\begin{array}{c}\text { Poids des animaux } \\
(\mathrm{g})\end{array}$ & $\begin{array}{c}\text { Poids relatifs des foies } \\
(\mathrm{g} / 100 \mathrm{~g} \text { de rat) }\end{array}$ \\
\hline Maïs & 390 & 2,90 \\
Maïs + cholestérol & 399 & 4,45 \\
Coprah & 395 & 3,04 \\
Coprah + cholestérol & 405 & 3,72 \\
Ecart-type commun sur la moyenne & 7,8 & 0,064 \\
\hline & \multicolumn{1}{c}{ Analyse statistique (F de Fisher) } \\
\hline Interaction & $0,001 \mathrm{NS}$ & (1) $45,6^{* *}$ \\
Effet huile & 0,4 NS & (2) $65,3^{* *}$ \\
Effet cholestérol & $1,6 \mathrm{NS}$ & (3) $293,5^{* *}$ \\
& & (4) $57,3^{* *}$ \\
\hline
\end{tabular}

Comparaisons par rapport à (1) régime sans cholestérol, (2) régime avec cholestérol, (3) régime avec maïs et (4) régime avec coprah. 
2) Protéines et cholestérol de la fraction microsomale. - Lorsque le cholestérol est présent dans le régime, on observe un effet de l'huile sur la teneur du foie en protéines microsomales (tabl. 3). En effet, celle-ci est plus élevée avec I'huile de coprah qu'avec l'huile de maïs : l'augmentation est de $26 \%$. Par contre, les quantités de protéines microsomales par foie entier ne sont affectées que par la présence de cholestérol alimentaire. Dans ce cas, les quantités totales de protéines microsomales sont supérieures de $40 \%$ pour les régimes comportant du cholestérol par rapport aux régimes n'en comportant pas.

Les facteurs étudiés sont sans effet sur les teneurs des foies en cholestérol microsomal (tabl, 3). Cependant, comme dans le cas des protéines, la présence de cholestérol alimentaire a un effet stimulant sur les quantités totales de cholestérol microsomal (53\% d'augmentation), résultant essentiellement de l'augmentation de la taille des foies.

\section{TABLEAU 3}

Protéines et cholestérol de la fraction microsomale (moyennes et écart-type commun, 8 rats/lot)

\begin{tabular}{|c|c|c|c|c|}
\hline \multirow{2}{*}{ Traitements } & \multicolumn{2}{|c|}{ Protéines } & \multicolumn{2}{|c|}{ Cholestérol } \\
\hline & $\mathrm{mg} / \mathrm{g}$ de foie & $\mathrm{mg} /$ foie entier & $\mathrm{mg} / \mathrm{g}$ de foie & $\mathrm{mg} /$ foie entier \\
\hline \multirow{6}{*}{$\begin{array}{l}\text { Maïs } \\
\text { Maïs + cholestérol } \\
\text { Coprah } \\
\text { Coprah + cholestérol } \\
\text { Ecart-type commun sur la } \\
\text { moyenne }\end{array}$} & 28,7 & 323 & 0,54 & 6,1 \\
\hline & 26,1 & 465 & 0,47 & 8,3 \\
\hline & 30,0 & 361 & 0,49 & 5,9 \\
\hline & 32,8 & 495 & 0,66 & 10,1 \\
\hline & 0,99 & 18,8 & 0,065 & 0,99 \\
\hline & \multicolumn{4}{|c|}{ Analyse de variance ( $F$ de Fisher) } \\
\hline Interaction & $7,4^{*}$ & $0,04 \mathrm{NS}$ & $3,5 \mathrm{NS}$ & $1,0 \mathrm{NS}$ \\
\hline Effet huile & $\begin{array}{l}\text { (1) } 0,9 \mathrm{NS} \\
\text { (2) } 23,0^{* *}\end{array}$ & 3,2 NS & $1,2 \mathrm{NS}$ & $0,6 \mathrm{NS}$ \\
\hline Effet cholestérol & $\begin{array}{l}\text { (3) } 3,5 \mathrm{NS} \\
\text { (4) } 3,9 \mathrm{NS}\end{array}$ & $53,5^{* *}$ & $0,6 \mathrm{NS}$ & $10,2^{* *}$ \\
\hline
\end{tabular}

Comparaisons par rapport à (1) régime sans cholestérol, (2) régime avec cholestérol, (3) régime avec maïs et (4) régime avec coprah.

3) Teneurs en cytochrome $P 450$ et $b_{5}$ de la fraction microsomale et activités de la NADPH-cytochrome $c$ réductase et de la NADH-cytochrome c réductase. - Les différents traitements sont sans effet sur les teneurs des microsomes en cytochrome P450 (tabl. 4). Par contre, la présence de cholestérol dans les régimes entraîne une augmentation de $16 \%$ des concentrations microsomales en cytochrome $b_{5}$. En outre, le cholestérol alimentaire provoque une augmentation des stocks hépatiques en cytochrome P450 et $b_{5}$, due à l'augmentation de la taille des foies.

L'activité de la NADPH-cytochrome c réductase (tabl. 4) est modifiée, d'une part, suivant la nature de l'huile ingérée et d'autre part, par la présence de cho- 


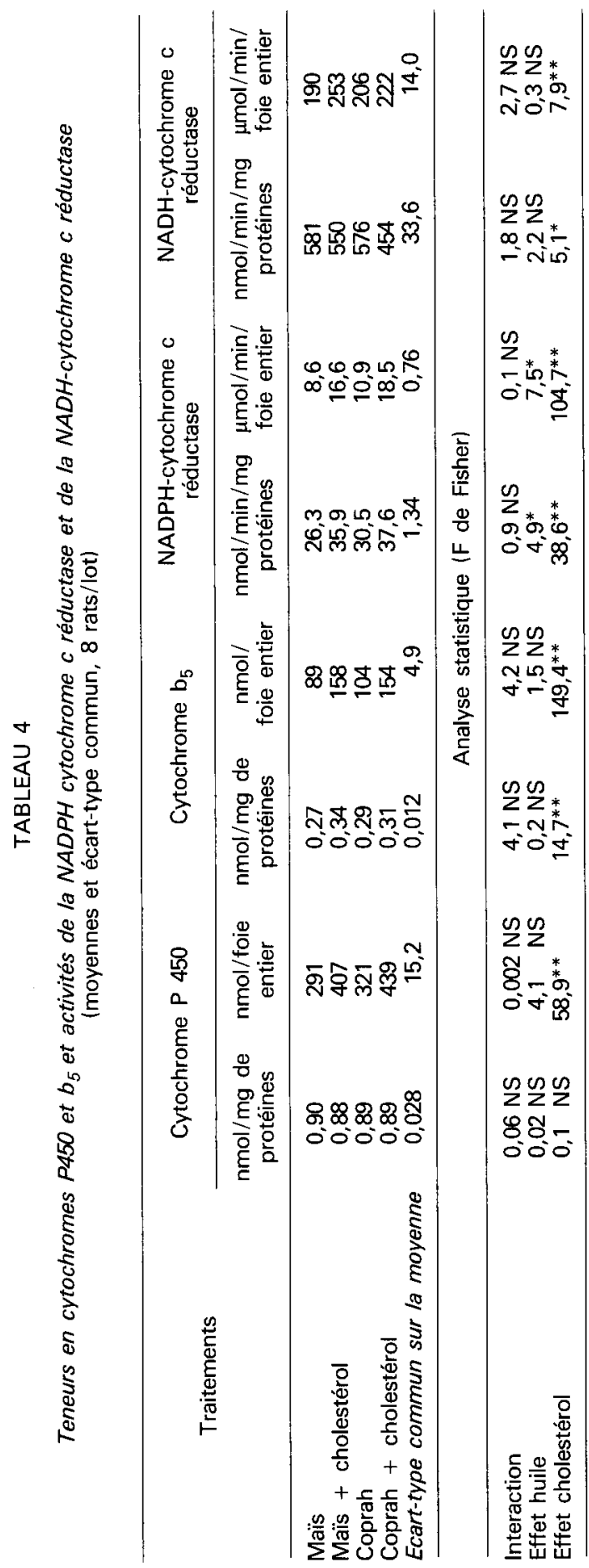


lestérol alimentaire. En effet, cette activité est légèrement supérieure chez les rats nourris avec des régimes renfermant de l'huile de coprah par rapport à celle des rats nourris avec des régimes renfermant de l'huile de maïs (10\% d'augmentation). D'autre part, cette activité augmente de $30 \%$ sous l'effet du cholestérol alimentaire. Par contre, en ce qui concerne l'activité spécifique de la NADHcytochrome $\mathrm{c}$ réductase, on observe une légère diminution due au cholestérol alimentaire $(13 \%)$.

4) Activités de l'aminopyrine déméthylase, de l'aniline hydroxylase et de la laurate hydroxylase (tabl. 5). - Les activités de l'aniline hydroxylase et de la laurate hydroxylase ne sont pas modifiées par les facteurs étudiés. Toutefois, la vitesse de déméthylation de l'aminopyrine est affectée par le type d'huile ingérée. Cette activité est supérieure de $34 \%$ chez des rats nourris à l'huile de maïs par rapport aux rats nourris à l'huile de coprah. Lorsque l'on exprime ces activités par foie entier, on constate une stimulation de l'aminopyrine déméthylase et de l'aniline hydroxylase sous l'effet du cholestérol alimentaire.

\section{Discussion et conclusion.}

De nombreux travaux établissent l'importance de l'influence des lipides alimentaires sur les monoxygénases microsomales dépendantes du cytochrome P450 (Hietanen et al., 1978 ; Wade et Norred, 1976). Des études ont été réalisées sur les effets de différents types d'acides gras ingérés, d'autres sur les effets de la présence de cholestérol dans le régime, mais à notre connaissance aucune n'a porté sur les effets des deux facteurs associés. Par ailleurs, Bochenek et Rodgers (1978) montrent que les effets du cholestérol alimentaire sur le métabolisme hépatique des acides gras et du cholestérol varient selon la nature (saturée ou insaturée) des acides gras du régime.

En ce qui concerne les effets dus au cholestérol alimentaire, Lang et Hänninen (1976) observent un effet stimulant de ce facteur. Des rats mâles Wistar nourris avec un régime standard commercial renfermant $4 \%$ de cholestérol ont des teneurs en cholestérol, phospholipides et protéines microsomales supérieures à celles de rats nourris avec le même régime ne comportant pas de cholestérol. Ils observent également une stimulation des activités de la NADPH-cytochrome c réductase, de la p-nitroanisole-O-déméthylase et de I'UDP-glucuronyl transférase. Ces auteurs attribuent ces variations d'activités à des modifications de la conformation des membranes microsomales, celles-ci étant mises en évidence à l'aide de molécules sondes fluorescentes. D'autre part, Hietanen et al. (1978) observent chez des rats mâles Wistar nourris avec un régime contenant $2 \%$ de cholestérol, une stimulation de certaines monoxygénases microsomales hépatiques (p-nitroanisole déméthylase, éthoxycoumarine dééthylase) par rapport à des rats nourris avec un régime identique mais ne renfermant pas de cholestérol. Cependant, ils n'observent ni élévation du taux microsomal en cytochrome P450, ni augmentation de l'activité de la NADPH-cytochrome c réductase. Nos propres résultats $n$ 'indiquent aucun effet stimulant du cholestérol sur les monoxygénases 


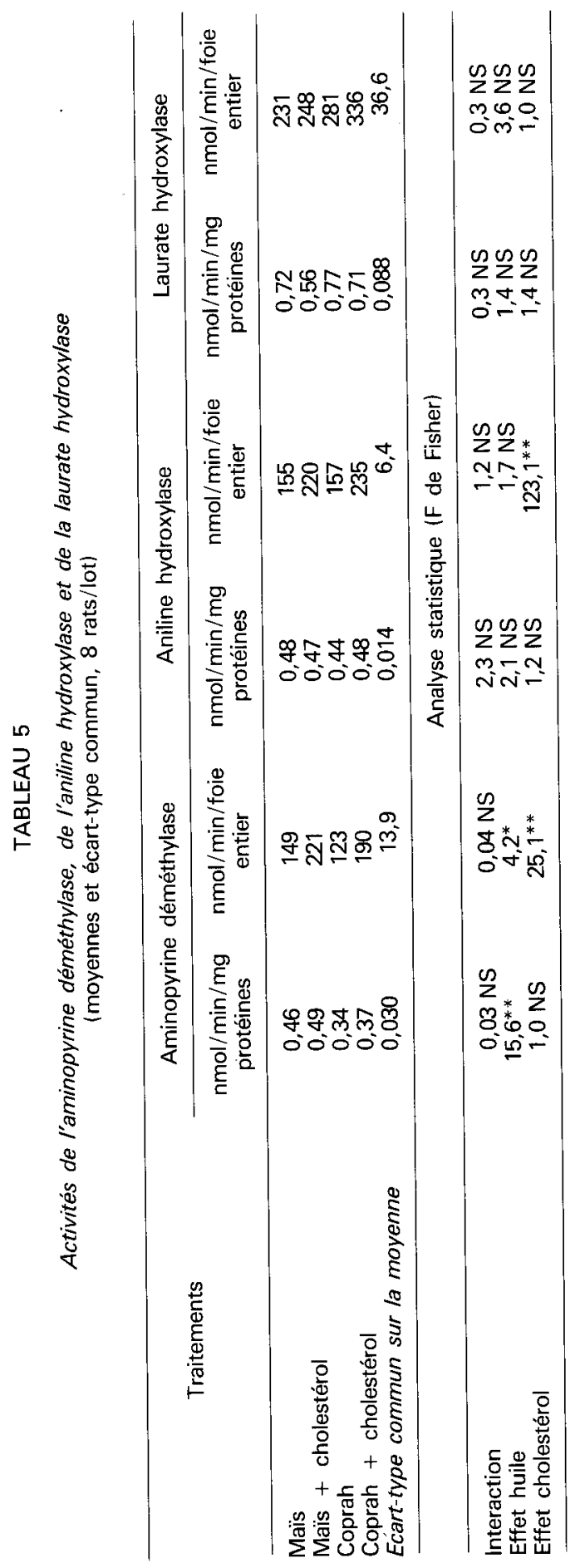


microsomales mesurées, à savoir aminopyrine déméthylase, laurate hydroxylase et aniline hydroxylase. Notons que les conditions expérimentales utilisées par ces auteurs divergent des nôtres quant à la durée du traitement (4 semaines au lieu de 9 semaines) et quant au pourcentage et la nature des lipides du régime $(3,5 \%$ de lipides au lieu de $15 \%)$. Par contre, nous constatons une élévation de l'activité de la NADPH-cytochrome c réductase chez des rats nourris avec des régimes comportant $2 \%$ de cholestérol, ainsi qu'une augmentation de $16 \%$ des teneurs en cytochrome $b_{5}$. Cette augmentation pourrait s'expliquer par le fait que ce cytochrome intervient non seulement au niveau des systèmes d'oxydation des substances xénobiotiques, mais joue également un rôle de transporteur d'électrons au niveau des systèmes de désaturation des acides gras (Okayasu et al., 1977 ; Oshino et al., 1971 ; Strittmatter et al., 1974). Or, comme l'observent Bochenek et Rodgers (1978), le cholestérol alimentaire provoque une demande accrue en acides gras polyinsaturés. II reste néanmoins à déterminer si les activités de désaturation sont réellement augmentées quand le cholestérol est présent dans le régime.

Le cholestérol alimentaire ne stimule aucune des autres activités enzymatiques mesurées. Néanmoins, les poids relatifs des foies étant fortement augmentés par ce facteur ( $22 \%$ à $53 \%$ d'augmentation suivant le type d'huile ingérée), on observe une augmentation de toutes les activités enzymatiques mesurées lorsque celles-ci sont exprimées par foie entier et non plus par mg de protéines microsomales. De plus, les stocks hépatiques en protéines et cholestérol microsomaux sont également augmentés par l'apport en cholestérol alimentaire.

Les variations du type d'huile ingérée ne produisent pas un effet uniforme sur l'activité des différentes enzymes microsomales. En effet, les teneurs microsomales en cytochrome $\mathrm{P} 450$ et $\mathrm{b}_{5}$ ne varient pas suivant la nature de l'huile. L'activité de la NADPH-cytochrome $c$ réductase est légèrement augmentée (10\%) chez les rats ayant ingéré de I'huile de coprah par rapport à ceux ayant ingéré de l'huile de maïs. En ce qui concerne les trois activités enzymatiques dépendantes du cytochrome P450, que nous avons déterminées, on remarque une augmentation de l'activité de l'aminopyrine déméthylase chez les rats ayant ingéré de I'huile de maïs par rapport aux rats ayant ingéré de I'huile de coprah. Les deux autres activités, laurate hydroxylase et aniline hydroxylase ne varient pas. L'aminopyrine et le laurate sont des substrats de type I pour le cytochrome P450 alors que l'aniline est un substrat de type II. Or Agradi et al. (1975) montrent que chez des rats nourris avec un régime comportant $10 \%$ de graisses saturées, il y a réduction de l'activité de la benzopyrène hydroxylase sans réduction concomitante de l'aniline hydroxylase, par rapport à des rats nourris avec un régime renfermant $10 \% d^{\prime}$ 'huile d'olive. Ces auteurs suggèrent que les effets différents du type d'huile consommée sur les activités de type I et celles de type II, sont dus principalement au taux d'acide linoléique présent dans les huiles $10,07 \%$ des calories dans le cas de l'huile saturée et 1,3\% des calories dans le cas de l'huile d'olive). Un apport insuffisant en acides gras essentiels modifie les activités de type I mais n'affecte pas celles de type II. D'autre part, Caster et al. (1970) montrent que le maximum d'activité de l'aniline hydroxylase est atteint lorsque l'acide linoléique représente $0,15 \%$ des calories du régime, alors que le 
maximum d'activité est atteint pour l'oxydase de l'heptachlore, lorsque le linoléate représente $1 \%$ des calories du régime. Dans nos propres régimes, l'acide linoléique représente $18 \%$ des calories dans le cas de l'huile de maïs et $2 \%$ des calories dans le cas de l'huile de coprah. Nous pensons donc que les effets variables du type d'huile ingérée sur les activités de type I et de type II ne sont pas seulement imputables au taux d'acide linoléique de I'huile, mais que d'autres facteurs tels que le degré d'insaturation ou la longueur de chaîne des acides gras constituants des huiles interviennent. De plus, tous les substrats de type I ne sont pas affectés de la même manière par le type d'huile ingérée puisque l'on observe une variation d'activité pour l'aminopyrine déméthylase mais non pour la laurate hydroxylase. En ce qui concerne cette dernière activité, nous remarquons que malgré les pourcentages en acide laurique très différents dans les deux types d'huile ( $45 \%$ dans l'huile de coprah et $0 \%$ dans I'huile de maïs), nous n'observons pas de modifications de cette activité. Toutefois, la voie de I'whydroxylation des acides gras n'est pas très importante $d^{\prime}$ un point de vue métabolique et ne représente que $5 \%$ seulement de la voie classique de la $\beta$ oxydation (Björkhem, 1978).

Les deux facteurs étudiés n'interagissent sur aucune des activités enzymatiques mesurées. La présence de cholestérol dans les régimes, en revanche, provoque une augmentation des poids relatifs des foies qui est très différente suivant le type d'huile consommée. L'augmentation est en effet beaucoup plus importante chez les rats ayant ingéré I'huile de maïs. En ce qui concerne les teneurs des foies en protéines microsomales, la présence de cholestérol dans le régime fait apparaître une variation due au type d'huile ingérée. Nous n'avons pas déterminé les quantités de lipides des foies mais nous pensons que ces différences des poids relatifs des foies sont imputables, pour une partie au moins, à une augmentation des lipides totaux hépatiques. Bochenek et Rodgers (1978) montrent en effet que chez des rats nourris pendant 4 semaines avec un régime renfermant $1 \%$ de cholestérol et $10 \%$ de lipides neutres, les augmentations des lipides hépatiques dues à la présence de cholestérol sont de $23 \%$ lorsque l'huile du régime est à dominante saturée et de $65 \%$ quand l'huile du régime est à dominante insaturée.

Cette étude nous a donc permis de constater que les lipides alimentaires ne produisent pas un effet identique sur les activités de différentes monoxygénases microsomales. Nos résultats et ceux de la littérature nous amènent à penser que de multiples facteurs tels que le pourcentage de cholestérol, les pourcentages des différents acides gras, la longueur de chaîne et le degré d'insaturation de ces acides gras, affectent ces activités de manière variable.

Reçu en juin 1982.

Accepté en octobre 1982.

Remerciements. - Les auteurs remercient le Dr Salaün pour son aide apportée aux dosages de la laurate hydroxylase. 


\section{Références}

ABELL L. L., LEVY B. B., BRODIE B. B., KENDALL F. E., 1952. A simplified method for the estimation of total cholesterol and demonstration of its specificity. J. biol. Chem., 195, 357-366.

AGRADI E., SPAGNUOLO C., GALLI C., 1975. Dietary lipids and aniline and benzopyrene hydroxylations in liver microsomes. Pharmacol. Res. Com., 7, 469-480.

BJÖRKHEM I., 1978. On the quantitative importance of $\omega$-oxidation of fatty acids. J. Lipid Res., 19, 585-590.

BOCHENEK W., RODGERS J. B., 1978. Effects of satured and unsatured fats given with or without dietary cholesterol on hepatic cholesterol synthesis and hepatic lipid metabolism. Biochim. biophys. Acta, 528, 1-16.

CASTER W. O., WADE A. E., GREenE F. E., MEADOWS J. S., 1970. Effect of different levels of corn oil in the diet upon the rate of hexobarbital, heptachlor and aniline metabolism in the liver of the male white rat. Life Sci, 9, 181-190.

CENTURY B., 1973. A role of the dietary lipid in the ability of phenobarbital to stimulate drug detoxification. J. Pharmac. exp. Ther., 185, 185-194.

DAGNÉLIE P., 1973. Théorie et méthodes statistiques. Les Presses Agronomiques, Gembloux.

GILBERT D., GOLBERG L., 1965. Liver response tests. III. Liver enlargment and stimulation of microsomal processing enzyme activity. Fd. Cosmet. Toxicol., 3, 417-432.

GLAUMAN H., DALLNER G., 1968. Lipid composition and turn-over of rough and smooth microsomal membranes in rat liver. J. Lipid Res., 9, 720-729.

HAMMER C. T., WILLS E. D., 1979. The effect of dietary fats on the composition of the liver endoplasmic reticulum and oxidative drug metabolism. Br. J. Nut., 41, 465-475.

HIETANEN E., HÄNNINEN O., LAITINEN M., LANG M., 1978. Dietary cholesterol-induced enhancement of hepatic biotransformation rate in male rats. Pharmacology, 17, 163-172.

HUBBEL R. B., MENDEL L. B., WAKEMAN A. J., 1937. New salt mixture for use in experimental diets. J. Nut., 14, 273-278.

IMAI Y., ITO A., SATO R., 1966. Evidence for biochemically different types of vesicles in hepatic microsomal fraction. J. Biochem., 60, 417-428.

LAITINEN M., 1976. Enhancement of hepatic drug metabolism with dietary cholesterol in the rat. Acta pharmacol. toxicol., 39, 241-249.

LAMBERT L., WILLS E. D., 1977. The effect of dietary lipids peroxides, sterols and oxidised sterols on cytochrome P450 and oxidative demethylation in the endoplasmic reticulum. Biochem. Pharmac, 26, 1417-1421.

LANG M., HÄNNINEN O., 1976. Alterations in the activities of drug metabolism enzymes in rat liver microsomes by dietary and in vitro incorporated cholesterol, 595-604. In ULLRICH V., ROOTS I., HIDELBRANDT A., ESTABROOK R. Microsomes and drug oxidations, Pergamon Press, Oxford.

LANG M., LAITINEN M., HIETANEN E., VAINO H., 1976. Modification of microsomal membrane components and induction of hepatic drug biotransformation in rats on a high cholesterol diet. Acta pharmacol. toxicol., 39, 273-288.

LOWRY O. H., ROSEBROUGH N. J., FARR A. L., RANDALL R. J., 1951. Protein measurement with the Folin phenol reagent. J. biol. Chem., 193, 265-275.

MARSHALL W. J., McLEAN A. E. M., 1971. A requirement for dietary lipids for induction of cytochrome P450 by phenobarbitone in rat liver microsomal fraction. Biochem. J., 122, 569-573.

OKAYASU T., ONO T., SHINOJIMA K., IMAI Y., 1977. Involvement of cytochrome $b_{5}$ in the oxidative desaturation of linoleic acid to $\gamma$ linolenic acid in rat liver microsomes. Lipids, 12, 267-271.

OMURA T., SATO R., 1964. The carbon monoxide-binding pigment of liver microsomes. I. Evidence for its hemoprotein nature. J. biol. Chem., 239, 2370-2378.

OSHINO N., IMAI Y., SATO R., 1971. A function of cytochrome $b_{5}$ in fatty acid desaturation by rat liver microsomes. J. Biochem., 69, 155-167. 
PHILLIPS A. H., LANGDON R. G., 1962. Hepatic triphosphopyridine nucleotide-cytochrome c reductase : Isolation, characterization and kinetic studies. J. biol. Chem., 237, 2652-2660.

ROWE L., WILLS E. D., 1976. The effect of dietary lipids and vitamin E on lipid peroxide formation, cytochrome $\mathrm{P} 450$ and oxidative demethylation in the endoplasmic reticulum. Biochem. Pharmac., 25, 175-179.

SALAÜN J. P., BENVENISTE I., REICHHART D., DURST F., 1981. Induction and specificity of a (cytochrome $\mathrm{P} 450$ ) - dependent laurate in-chain-hydroxylase from higher plant microsomes. Eur. J. Biochem., 119, 651-655.

SPECTOR A. A., KADUCE T. L., DANE R. W., 1980. Effect of dietary fat saturation on acylcoenzyme A : cholesterol acyltransferase activity of rat liver microsomes. J. Lipid Res., 21, 169-179.

SRITTMATTER P., SPATZ L., CORCORAN D., RODGERS M. J., SETLOW B., REDLINE R., 1974. Purication and properties of rat liver microsomal stearyl coenzyme A desaturase. Proc. nat. Acad. Sci. USA, 71, 4565-4569.

WADE A. E., NORRED W. P., 1976. Effect of dietary lipid on drug-metabolizing enzymes. Fed. Proc., 35, 2475-2479. 